\title{
Onset of angioedema following a viper bite: different options for diagnostic procedure in an emergency department
}

\author{
Alessandro Riccardi, Pierangela Minuto, Anna Da Col, Viviana Panunzio*, Roberto Lerza \\ SC Pronto Soccorso e Medicina e Chirurgia d'Accettazione e d'Urgenza, Ospedale San Paolo, Savona, Italy \\ *SC Immunoematologia e Medicina Trasfusionale, ASL 2 Savonese, Savona, Italy
}

\section{ABSTRACT}

The unexpected onset of an angioedema in a patient observed in the emergency department after a viper bite, gave the opportunity to consider some different etiopathogenetic hypothesis and to discuss the most correct diagnostic procedure. The patient was a 60-year-old man who was treated with sartans for hypertension and reported a recent diag-

\section{Introduction}

Angioedema is a life-threatening clinical condition because of the mucosal swelling of the upper airways which may impair breathing. Face, neck, limbs, genitals and the gastrointestinal mucosa can be involved by a recurrent unpredictable oedema. The pathophysiology of allergic angioedema recognizes a histamine-mediated mechanism, and consequently a pruritic oedema and urticaria are typically detected. On the contrary, non-allergic angioedema symptoms are generally mediated by a bradykinin release, and urticaria and/or inflammatory and pruritic swelling are classically absent. Five different types of non-allergic angioedema are defined in literature (1). They include hereditary, acquired, idiopathic, pseudo-allergic, and renin-angiotensin-aldosteron system blocker-induced angioedema. Among these, acquired angioedema is considered a rare condition $(2,3)$; however, its incidence is probably underestimated and frequently unrecognized. The pathophysiology of this condition reflects a non-genetic C1-esterase inhibitor (C1-INH) defective activity due to decreased plasma levels or impaired function (1). Here we present a case of a possibly acquired angioedema, which was an interesting opportunity for the emergency physicians to discuss the different hypothesis of the diagnostic procedure

\section{Case report}

A 64-years-old man was seen for the first time in our emergency room in September 2010 because of a viper bite involving the fifth finger of the left foot. He had had a history of hypertension treated with angiotensin II receptor antagonist (candesartan) for approximately ten years. He reported a recent diagnosis of monoclonal gammopathy of uncertain significance (MGUS) IgMk made by a haematologist, and he had been submitted to many allergologic tests because in the last two years he had experienced sporadic and transient episodes, characterized by stuffy nose and swelling in the palpebral region. The checks results were negative and no specific agent had been found as precipitating factor. When admitted in the emergency room, he denied any known allergy. No physician had seen and visited the patient during the described episodes. At the time of admission he appeared in good general conditions, his physical examination was inexpressive, the vital parameters and the basic laboratory findings were normal. A slight haematoma was present at the fifth finger of the left foot together with small signs from the snake bites. The patient had killed the snake and taken it to the hospital. nosis of monoclonal gammopathy of uncertain significance. Based on the patient history and on the clinical aspects of the angioedema, the authors consider the possibility of an acquired non-allergic angioedema and its probable close relationship with a lymphoproliferative disorder. Keywords: angioedema, viper bite, emergency department.

Among the first adopted measures antimicrobial prophylaxis with amoxicilline/clavulanate was started and antitetanus gammaglobulins were administered. The patient was then admitted to our ward for a short time of observation. After ten hours from his admission, during the night, the patient showed a sudden onset of a non-inflammatory oedema of the face, in particular of the palpebral region and lips (fig. 1a, b), and of the hands. Legs, foot and genitals were not involved. Breathing and vital parameters were not impaired, there was however only a slight involvement of the mucosa of tongue and throat. Then steroids, antihistamines and epinephrine were administered intravenously. All symptoms fully disappeared without complications within 24 hours. The patient told physicians that this episode was similar to those he had periodically complained in the last two years. A direct relationship between the snake venom and the onset of angioedema was excluded by the consultant of the Anti-poison Centre (Fondazione Maugeri-Pavia). The viper bite had only locally induced a slight haematoma confined to the upper part of the left foot (fig. 2).

The patient was discharged about 48 hours after the admission, and he was addressed to a further haematological and allergologic evaluation with the aim to reconsider the diagnosis of MGUS and to perform specific immunological tests. The latter confirmed the presence of a serum monoclonal paraprotein IgMk and showed the positivity of the Bence Jones protein. The value of serum IgM was increased (1182 mg/dl normal range 47-230), IgA were 139 $\mathrm{mg} / \mathrm{dl}$ (90-395) and IgG $696 \mathrm{mg} / \mathrm{dl}$ (840-1660). The level of the $\mathrm{C} 1$-inhibitor $(\mathrm{C} 1-\mathrm{INH})$ resulted at the lowest level of the normal range. This is possible because there is a report of a lymphomaassociated angioedema with normal plasma concentration of C1-INH (4) and in lymphoproliferative disease it is hypothized that auto-antibodies to $\mathrm{C} 1-\mathrm{INH}$ make the protein functionally inactive or increase its catabolism (3). The $\mathrm{M}$ component might correspond to the anti-C1-INH auto-antibodies (5). To definitely confirm a diagnosis of acquired angioedema, reduced $\mathrm{Clq}$ molecule levels, normal level of $\mathrm{C} 3$ and a low $\mathrm{C} 4$ should be detected together with the demonstration of a decreased C1-INH plasma concentration or activity, but only few centers are able to give reliable results.

Finally, the bone marrow biopsy showed the presence of a small monoclonal population of B lymphocytes. Phenotypically the cells expressed CD5, CD19, CD20, CD22, were negative for CD10, $\mathrm{CD} 23$ and restricted for $\mathrm{k}$ chains in a picture suggestive for a mature B cells lymphoproliferative disorder. 

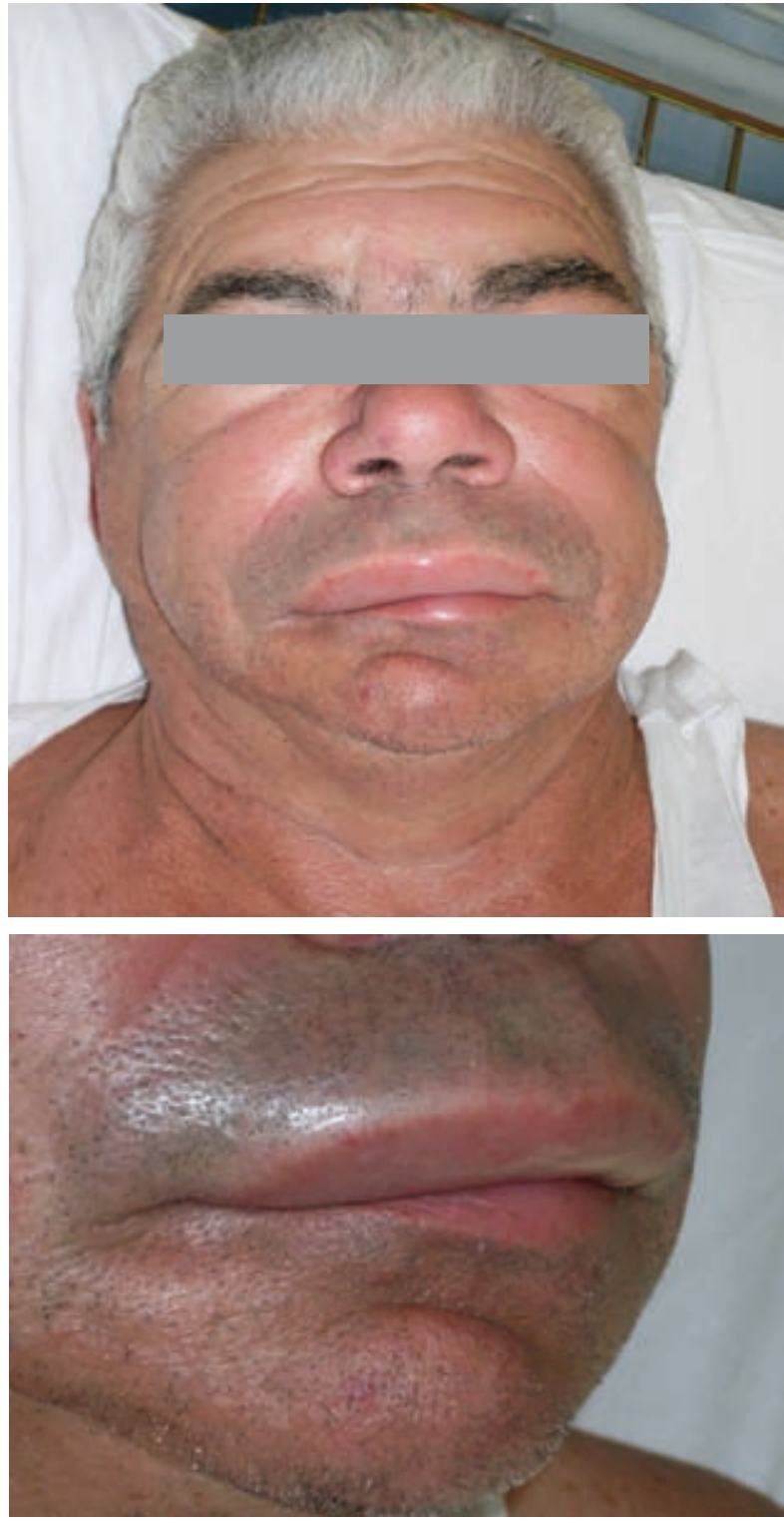

Fig. 1 - Non-inflammatory oedema of the face (a) and lips (b)

\section{Discussion}

Facing the onset of an angioedema physicians should ask themselves some questions. First of all, it is important to establish if the angioedema has an allergic or a non-allergic cause. The snake poison, the administration of antibiotics and gammaglobulin as anti-tetanus prophylaxis could theoretically be antigens inducing a histamine-mediated allergic angioedema. However, in our case this appears an unlikely etiopathogenetic hypothesis, because the patient complained recurrent episodes of angioedema in the last two years apart from the exposure to specific agents. Moreover, the Anti-poison Centre excluded a relationship between the viper venom and the angioedema, and literature reports only of some cases of angioedema following specific antivento treatment (6). We agree with the Anti-poison Centre, but trying to explain the coincidence between the viper bite and the episode of angioedema is suggestive to consider the snake bite as a precipitating stressing event. In fact, the old term "angioneurotic oedema" derived from the opinion that in non-allergic angioedema precipitating factors included minor trauma, estrogen, infection and emotional stress
$(7,8)$. According to this statement, a snake bite can be considered a minor trauma that certainly induces an emotional stress.

In our patient two aspects were of importance in addressing the diagnosis toward a non-allergic angioedema. The first one was the absence of anamnestic data suggestive for a known allergy: allergologic cutaneous and blood tests previously carried out resulted negative. The second one is the typical non-inflammatory cutaneous swelling that wasn't itchy or painful. The lack of signs of urticaria further support the hypothesis of a non-allergic angioedema (1). Among the different types of the latter condition a hereditary angioedema seems unlikely due to the absence of family history, the age of the patient and the relatively recent time of beginning of the disorder. It is well known that angioedema can be an important side effect not only of the angiotensin-converting enzyme inhibitors therapy, but also of a treatment with sartans (9-11). We think it was correct to advise the patient to stop the drug even if he had taken sartans for more than ten years, and then a correlation with the appearance of angioedema did not seem believable.

On the basis of the considerations reported so far, we were more and more suspicious of a possible close relationship between the MGUS and the angioedema. In our patient both the first episode of angioedema and the diagnosis of MGUS were approximately of the same period. Moreover, monoclonal gammopathies, lymphoproliferative syndromes, autoimmune diseases, neoplasms and infections have been reported as diseases associated with acquired angioedema $(3,12)$. Although not frequently reported, lymphoproliferative disorders of mature B-lymphocytes expressing IgM kappa or lambda are the prominent disorders underlying an acquired angioedema $(3,13-15)$, and the latter is an important, potentially life-threatening complication of lymphoma. It is also important a prompt suspicion and recognition of these conditions, because an appropriate treatment of the associated disease could resolve angioedema in some patients (3). Obviously, in an emergency department there is no possibility to study these cases deeply from a haematological and immunological point of view, but it's important to think of this possible association and to correctly address the patient to a diagnostic procedure. In an emergency setting it is not unusual to see repeatedly the same patient with episodic angioedema, but often the emergency physicians are not involved and informed of the final diagnosis, while their role is limited to the surveillance of the vital functions and the observation of patients who frequently have a self-limiting disturbance. We were extremely satisfied by the fact that, in the presented case, the emergency physician was the first one to hypothize the correct diagnosis and to put haematologist and allergologist in contact, in order to consider the close relationship between the two conditions they had separately evaluated.

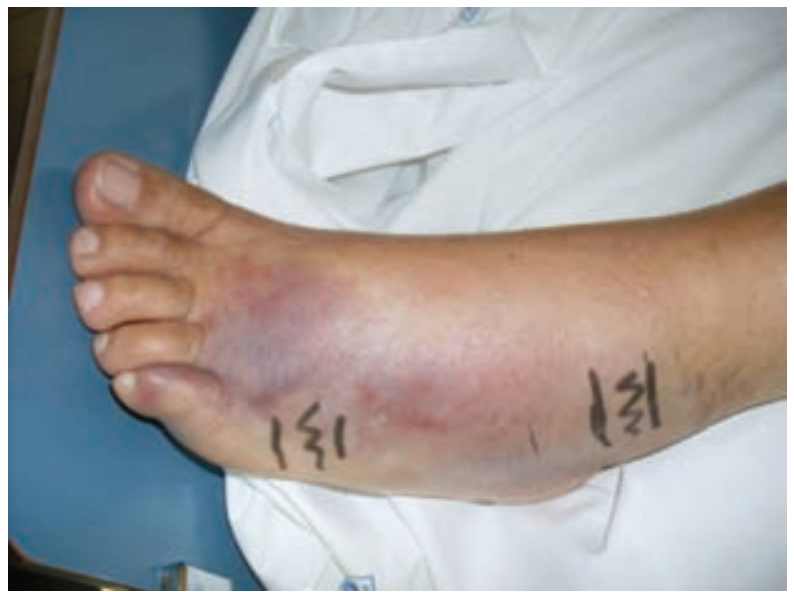

Fig. 2 - Haematoma of the left foot induced by the viper bite. 


\section{References}

1. Bas M, Adams V, Suvorava T, Niehues T, Hoffmann TK, Kojda G. Non allergic angioedema: role of bradykinin. Allergy 2007;62:842-56.

2. Zingale LC, Castelli R, Zanichelli A, Cicardi M. Acquired deficiency of the inhibitor of the first complement component: presentation, diagnosis, corse and conventional management. Immunol Allergy Clin North Am 2006;26:669-90.

3. Cicardi M, Zanichelli A. Acquired angioedema. Allergy Asthma \& Clin Immunol 2010;6:1492-96.

4. Gaur S, Cooley J, Aish L, Weinstein R. Lymphoma-associated paraneoplastic angioedema with normal $\mathrm{C} 1$-inhibitor activity : does danazol work? Am J Hematol 2004;77:296-98.

5. Cicardi M, Beretta A, Colombo M, Gioffre D, Cugno M, Agostoni A. Relevance of lymphoproliferative disorders and anti-C1 inhibitor autoantibodies in acquired angioedema. Clin Exp Immunol 1996;106:475-80.

6. Stahel E, Wellauer R, Freyvogel TA. Poisoning By domestic viper (vipera berus and vipera aspis). A retrospective study of 113 patients. Schweitz Med Wochenschr 1985;115:890-96.

7. Baxi S, Dinakkar C. Urticaria and angioedema. Immunol Allergy Clin North Am 2005;25:353-67.

8. Fay A, Abinum M. Current management of hereditary angioedema. J Clin Pathol 2002;55:266-70.

9. Roskiewicz F, Andriamanana I, Gras-Champel V, Andrejak
M, Massy ZA. Iatrogenic angioedema: the role of angiotensin converting enzyme inhibitor and angiotensin II receptor blockers. Nephrol Ther 2007;3:89-95.

10. Malde B, Regalado J, Greenberger PA. Investigation of angioedema associated with the use of angiotensin-converting enzyme inhibitors and angiotensin receptor blokers. Ann Allergy Asthma Immunol 2007;98:57-63.

11. Haymore BR, Yoon J, MikitaCP, Klote MM, DeZee KJ. Risk of angioedema with angiotensin receptor blockers in patients with prior angioedema associated with angiotensin-converting enzyme inhibitors: a meta-analysis. Ann Allergy Asthma Immunol 2008;101:495-9.

12. Nettis E, Colanardi MC,Loria MP, Vacca A. Acquired C1 inhibitor deficiency in a patient with systemic lupus erythematosus: a case report and review of the literature. Eur J Clin Invest 2005;35:781-4.

13. Bain BJ, Catovsky D, Ewan PW. Acquired angioedema as a presenting feature of lymphoproliferative disorders of mature B-Lymphocytes. Cancer 1993;72:3318-22.

14. Bibi-Triki T, Eclache V, Frilay Y, Stirnemann J, FremeauxBacchi V, Fain O. Acquired C1 inhibitor deficiency associated with lymphoproliferative disorders: four cases. Rev Med Interne 2004;25:667-72.

15. Wellwood J, Taylor K, Wright S, Bentley M, Eliadis P. Angioedema in the emergency department: a presentation of lymphoma. Emerg Med (Fremantle) 2001;13:465-8 